PAPERS BY AUTHOR

\title{
GETTING STARTED
}

TRADEMARKS

SEARCH

Published by

(D) computer
society

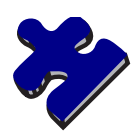

Reengineering Forum

CORPORATE SPONSORS

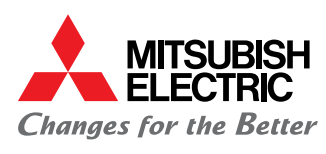

Microsoft

NTTDaTa

Global IT Innovator

\section{SUPPORTERS}

IPA $\begin{gathered}\text { Betertifio } \\ \text { winh }\end{gathered}$

Graduate School of

Information Science and Technology OSAKA UNIVERSITY

\section{I] Information Processing \\ Society of Japan}

Special Interest Group on Software Engineering

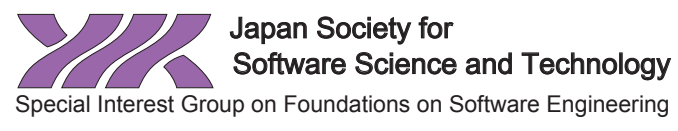

The Telecommunications Advancement Foundation

Support Center for Advanced Telecommunications Technology Research Foundation 


\section{IEEE 23rd International Conference on Software Analysis, Evolution, and Reengineering}

14-18 March 2016

Osaka, Japan 


\section{IEEE 23rd International Conference on Software Analysis, Evolution, and Reengineering \\ SANER 2016 \\ Table of Contents \\ Volume - 1}

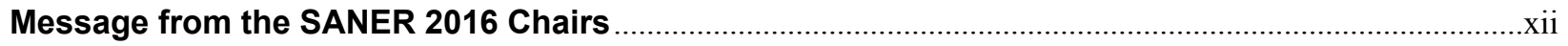

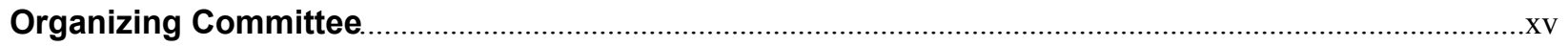

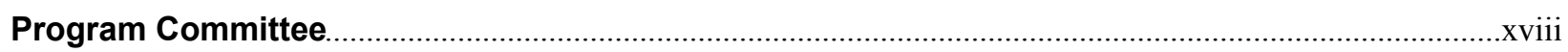

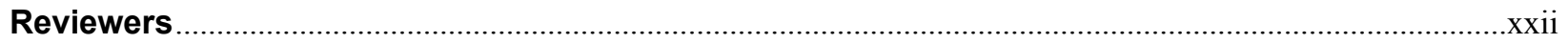

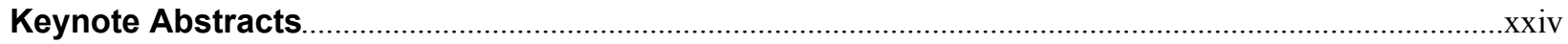

Sponsors

\section{Main Research}

\section{Refactoring}

Domino Effect: Move More Methods Once a Method is Moved

Hui Liu, Yuting Wu, Wenmei Liu, Qiurong Liu, and Chao Li

Supporting Selective Undo for Refactoring

Xiao Cheng, Yuting Chen, Zhenjiang Hu, Tao Zan, Mengyu Liu, Hao Zhong,

and Jianjun Zhao

Finding the Best Compromise Between Design Quality and Testing Effort During

Refactoring

Rodrigo Morales, Aminata Sabané, Pooya Musavi, Foutse Khomh, Francisco Chicano,

and Giuliano Antoniol

Studying the Relation between Anti-Patterns in Design Models and in Source Code

Bilal Karasneh, Michel R. V. Chaudron, Foutse Khomh, and Yann-Gaël Guéhéneuc

\section{Clones}

An Empirical Study on Recommendations of Similar Bugs

Henrique Rocha, Marco Tulio Valente, Humberto Marques-Neto, and Gail C. Murphy 
Cross-Architecture Binary Semantics Understanding via Similar Code Comparison Yikun Hu, Yuanyuan Zhang, Juanru Li, and Dawu Gu

Bug Replication in Code Clones: An Empirical Study 68 Judith F. Islam, Manishankar Mondal, and Chanchal K. Roy

On the Relationship of Inconsistent Software Clones and Faults: An Empirical Study Stefan Wagner, Asim Abdulkhaleq, Kamer Kaya, and Alexander Paar

\section{People}

Software-Specific Named Entity Recognition in Software Engineering Social Content .90

Deheng Ye, Zhenchang Xing, Chee Yong Foo, Zi Qun Ang, Jing Li, and Nachiket Kapre

Forking and the Sustainability of the Developer Community Participation - An

Empirical Investigation on Outcomes and Reasons Ayushi Rastogi and Nachiappan Nagappan

More Common Than You Think: An In-depth Study of Casual Contributors

Gustavo Pinto, Igor Steinmacher, and Marco Aurélio Gerosa

A Study of Visual Studio Usage in Practice .....

Sven Amann, Sebastian Proksch, Sarah Nadi, and Mira Mezini

\section{New Sources}

Evaluating Automatic Spreadsheet Metadata Extraction on a Large Set of Responses

from MOOC Participants .....

Sohon Roy, Felienne Hermans, Efthimia Aivaloglou, Jos Winter, and Arie van Deursen

Localizing Multiple Faults in Simulink Models

Bing Liu, Lucia, Shiva Nejati, Lionel Briand, and Thomas Bruckmann

A More Accurate Model for Finding Tutorial Segments Explaining APIs

He Jiang, Jingxuan Zhang, Xiaochen Li, Zhilei Ren, and David Lo

An Empirical Study on the Use of CSS Preprocessors 168

Davood Mazinanian and Nikolaos Tsantalis

\section{Quality}

Examining the Impact of Self-Admitted Technical Debt on Software Quality

Sultan Wehaibi, Emad Shihab, and Latifa Guerrouj

The Impact of Human Discussions on Just-in-Time Quality Assurance: An Empirical

Study on OpenStack and Eclipse

Parastou Tourani and Bram Adams

Generalizing the Analysis of Evolutionary Coupling for Software Change Impact

Analysis 201

Thomas Rolfsnes, Stefano Di Alesio, Razieh Behjati, Leon Moonen, and Dave W. Binkley 
History Driven Program Repair

Xuan Bach D. Le, David Lo, and Claire Le Goues

\section{Instrumental}

Negative Effects of Bytecode Instrumentation on Java Source Code Coverage .225

Dávid Tengeri, Ferenc Horváth, Arpád Beszédes, Tamás Gergely, and Tibor Gyimóthy

UROBOROS: Instrumenting Stripped Binaries with Static Reassembling

Shuai Wang, Pei Wang, and Dinghao Wu

Towards Transparent Introspection

Kevin Leach, Chad Spensky, Westley Weimer, and Fengwei Zhang

Linvail: A General-Purpose Platform for Shadow Execution of JavaScript

Laurent Christophe, Elisa Gonzalez Boix, Wolfgang De Meuter, and Coen De Roover

\section{PoLemic}

Custom-Tailored Variability Mining for Block-Based Languages

David Wille, Sandro Schulze, Christoph Seidl, and Ina Schaefer

A Case Study on Type Hints in Method Argument Names in Pharo Smalltalk Projects

Boris Spasojević, Mircea Lungu, and Oscar Nierstrasz

Visualizing Data-Flows in Functional Programs

Tobias Weck and Matthias Tichy

Tracking Null Checks in Open-Source Java Systems

Haidar Osman, Manuel Leuenberger, Mircea Lungu, and Oscar Nierstrasz

\section{IR-onic Mining}

Parameterizing and Assembling IR-Based Solutions for SE Tasks Using Genetic

Algorithms

Annibale Panichella, Bogdan Dit, Rocco Oliveto, Massimiliano Di Penta,

Denys Poshyvanyk, and Andrea De Lucia

Examining the Stability of Logging Statements

Suhas Kabinna, Weiyi Shang, Cor-Paul Bezemer, and Ahmed E. Hassan

Mining Analogical Libraries in Q\&A Discussions — Incorporating Relational

and Categorical Knowledge into Word Embedding

Chunyang Chen, Sa Gao, and Zhenchang Xing

RACK: Automatic API Recommendation Using Crowdsourced Knowledge

Mohammad Masudur Rahman, Chanchal K. Roy, and David Lo 


\section{Crystal Balls}

Do Developers Deprecate APIs with Replacement Messages? A Large-Scale Analysis

on Java Systems 360

Gleison Brito, Andre Hora, Marco Tulio Valente, and Romain Robbes

MICHAC: Defect Prediction via Feature Selection Based on Maximal Information

Coefficient with Hierarchical Agglomerative Clustering

Zhou Xu, Jifeng Xuan, Jin Liu, and Xiaohui Cui

On the Detection of Licenses Violations in the Android Ecosystem

Ons Mlouki, Foutse Khomh, and Giuliano Antoniol

Do Code Smells Impact the Effort of Different Maintenance Programming Activities?

Zéphyrin Soh, Aiko Yamashita, Foutse Khomh, and Yann-Gaël Guéhéneuc

\section{Mobile}

An Investigation into the Use of Common Libraries in Android Apps

Li Li, Tegawendé F. Bissyandé, Jacques Klein, and Yves Le Traon

Revisiting the Description-to-Behavior Fidelity in Android Applications

Le Yu, Xiapu Luo, Chenxiong Qian, and Shuai Wang

Mining Android Apps to Recommend Permissions

Md. Yasser Karim, Huzefa Kagdi, and Massimiliano Di Penta

Optimizing User Experience in Choosing Android Applications

Rubén Saborido, Giovanni Beltrame, Foutse Khomh, Enrique Alba, and Giuliano Antoniol

\section{Program Analysis}

Efficient and Precise Dynamic Slicing for Client-Side JavaScript Programs

Jiabin Ye, Cheng Zhang, Lei Ma, Haibo Yu, and Jianjun Zhao

Supporting Program Analysis for Non-Mainstream Languages: Experiences

and Lessons Learned

Andreas Grimmer, Florian Angerer, Herbert Prähofer, and Paul Grünbacher

Analyzing the State of Static Analysis: A Large-Scale Evaluation in Open Source

Software .

Moritz Beller, Radjino Bholanath, Shane Mcintosh, and Andy Zaidman

Marea: A Semi-Automatic Decision Support System for Breaking Dependency Cycles

Andrea Caracciolo, Bledar Aga, Mircea Lungu, and Oscar Nierstrasz 


\section{Eco-Logical}

When GitHub Meets CRAN: An Analysis of Inter-Repository Package Dependency

Problems

Alexandre Decan, Tom Mens, Maëlick Claes, and Philippe Grosjean

Achieving Knowledge Evolution in Dynamic Software Product Lines

Lorena Arcega, Jaime Font, Øystein Haugen, and Carlos Cetina

Haskell in Green Land: Analyzing the Energy Behavior of a Purely Functional

Language

Luís Gabriel Lima, Francisco Soares-Neto, Paulo Lieuthier, Fernando Castor,

Gilberto Melfe, and João Paulo Fernandes

Client-Side Energy Efficiency of HTTP/2 for Web and Mobile App Developers

Shaiful Alam Chowdhury, Varun Sapra, and Abram Hindle

\section{Release Engineering}

Predicting Build Co-changes with Source Code Change and Commit Categories

Christian Macho, Shane McIntosh, and Martin Pinzger

Release Practices for Mobile Apps - What do Users and Developers Think?

Maleknaz Nayebi, Bram Adams, and Guenther Ruhe

A Large Scale Study of Multiple Programming Languages and Code Quality

Pavneet Singh Kochhar, Dinusha Wijedasa, and David Lo

Botched Releases: Do We Need to Roll Back? Empirical Study on a Commercial Web

App

Noureddine Kerzazi and Bram Adams

\section{Early Research Achievements}

\section{ERA: APIs, Refactoring, and Design}

Parameter Values of Android APIs: A Preliminary Study on 100,000 Apps

Li Li, Tegawendé F. Bissyandé, Jacques Klein, and Yves Le Traon

Native or Web? A Preliminary Study on the Energy Consumption of Android

Development Models .....

Wellington Oliveira, Weslley Torres, Fernando Castor, and Bianca H. Ximenes

Composite Refactoring for Decoupling Multiple Classes

Yusuke Takahashi and Naoya Nitta

A Code Refactoring Dataset and Its Assessment Regarding Software Maintainability

István Kádár, Péter Hegedüs, Rudolf Ferenc, and Tibor Gyimóthy 
Frankencode: Creating Diverse Programs Using Code Clones

Hayley Borck, Mark Boddy, Ian J. De Silva, Steven Harp, Ken Hoyme, Steven Johnston,

August Schwerdfeger, and Mary Southern

Antipattern and Code Smell False Positives: Preliminary Conceptualization

and Classification

Francesca Arcelli Fontana, Jens Dietrich, Bartosz Walter, Aiko Yamashita, and Marco Zanoni

Identifying Utility Functions Using Random Forests

Tamara Mendes, Marco Tulio Valente, Andre Hora, and Alexander Serebrenik

\section{ERA: Mining and Empirical Studies}

Towards Building API Usage Example Metrics

Stevche Radevski, Hideaki Hata, and Kenichi Matsumoto

Software Language Identification with Natural Language Classifiers

Juriaan Kennedy van Dam and Vadim Zaytsev

At Ease with Your Warnings: The Principles of the Salutogenesis Model Applied

to Automatic Static Analysis

Jan-Peter Ostberg and Stefan Wagner

An Empirical Study on the Usage of the Swift Programming Language

Marcel Rebouças, Gustavo Pinto, Felipe Ebert, Weslley Torres, Alexander Serebrenik, and Fernando Castor

AutoBench: Finding Workloads That You Need Using Pluggable Hybrid Analyses

Yudi Zheng, Andrea Rosà, Luca Salucci, Yao Li, Haiyang Sun, Omar Javed,

Lubomir Bulej, Lydia Y. Chen, Zhengwei Qi, and Walter Binder

Analyzing the Decision Criteria of Software Developers Based on Prospect Theory

Kanako Kina, Masateru Tsunoda, Hideaki Hata, Haruaki Tamada, and Hiroshi Igaki

\section{Tool Demonstrations}

BUMPER: A Tool for Coping with Natural Language Searches of Millions of Bugs

and Fixes

Mathieu Nayrolles and Abdelwahab Hamou-Lhadj

BINSEC/SE: A Dynamic Symbolic Execution Toolkit for Binary-Level Analysis

Robin David, Sébastien Bardin, Thanh Dinh Ta, Laurent Mounier, Josselin Feist,

Marie-Laure Potet, and Jean-Yves Marion

CoreTAna: A Trace Analyzer for Reverse Engineering Real-Time Software Andreas Sailer, Michael Deubzer, Gerald Lüttgen, and Jürgen Mottok

Supporting Merge Conflict Resolution by Using Fine-Grained Code Change History 661

Yuichi Nishimura and Katsuhisa Maruyama 
Managing Traceability Links with MaTraca

Angela Lozano, Carlos Noguera, and Viviane Jonckers

Automated Generalization and Refinement of Code Templates with Ekeko/X

Tim Molderez and Coen De Roover

\section{Industrial Research}

Improving the Performance of a Large Scale Spreadsheet: A Case Study

Alaaeddin Swidan, Felienne Hermans, and Ruben Koesoemowidjojo

A Systematic Framework for Modernizing Legacy Application Systems

Timothy C. Fanelli, Scott C. Simons, and Sean Banerjee

Experience Report on Building ASTM Based Tools for Multi-language Reverse

Engineering

Günter Fleck, Wilhelm Kirchmayr, Michael Moser, Ludwig Nocke, Josef Pichler,

Rudolf Tober, and Michael Witlatschil

On Error-Class Distribution in Automotive Model-Based Software

Harald Altinger, Yanja Dajsuren, Sebastian Siegl, Jurgen J. Vinju, and Franz Wotawa

Designing and Developing Automated Refactoring Transformations: An Experience

Report

Gábor Szőke, Csaba Nagy, Rudolf Ferenc, and Tibor Gyimóthy

Author Index - Volume 1 .698 